\title{
Perfil dos Alunos Universitários dos Cursos de Educação Física e Fisioterapia em Relação à Alimentação e a Atividade Física
}

\section{Student Profile College Courses of Physical Education and Physical Therapy in Relation to Food and Physical Activity}

\author{
1 Josiane Vieira de Souza \\ ${ }^{1}$ Thaisnara Priscila Franco Bastos \\ ${ }^{2}$ Maria de Fatima Alves de Oliveira
}

\begin{abstract}
RESUMO
A regularidade na prática de atividade física e bons hábitos alimentares são idealizados como principais componentes de aspectos positivos relacionados à saúde. Devido à escassez de tempo, principalmente com a concomitância entre trabalho e estudo, universitários tendem a substituir as refeições de qualidade por lanches rápidos, além da pouca disposição para atividade física, o que nos despertou para o levantamento do presente estudo. Neste trabalho, analisou-se o perfil alimentar de universitários dos cursos de Educação Física e Fisioterapia de uma instituição privada de ensino superior situada na zona oeste do Estado do Rio de Janeiro. Participaram da pesquisa 129 estudantes, que pertenciam ao $2^{\circ}, 4^{\circ}, 6^{\circ}$ e $8^{\circ}$ períodos dos respectivos cursos. A coleta de dados foi feita através de um instrumento contendo questões objetivas relacionadas ao perfil, aos hábitos alimentares e atividade física. O instrumento foi aplicado no intervalo das aulas. Os dados revelaram que os estudantes do curso de Educação Física possuem hábitos alimentares e práticas de atividade física regulares, contradizendo alguns estudos sobre o tema.
\end{abstract}

Palavras-chave: hábitos alimentares; atividade física; universitários

\begin{abstract}
The regularity in the practice of physical activity and good eating habits are the main components of idealized positive aspects related to health. Because of the shortage of time, mainly with the concomitance between work and study, students tend to replace quality meals for snacks, in addition to the little disposition to physical activity, we woke up to the lifting of the present study. In this study, we analyzed food profiles of students of Physical Education and Physiotherapy courses of a private institution of higher education located in the West zone of Rio de Janeiro. Participants were 129 students, who belonged to the $2^{\text {nd }}$, $4^{\text {th }}$, $6^{\text {th }}$ and $8^{\text {th }}$ semesters of courses. Data collection was done through an instrument containing objective questions related to the profile, to eating habits and physical activity. The instrument was applied in the range of classes. The data revealed that the students of the course of Physical Education have eating habits and regular physical activity practices, contradicting some studies on the subject.
\end{abstract}

Keywords: eating habits; physical activity; university students

1 Estudantes de graduação pelo Centro Universitário Moacyr Sreder Bastos- UniMSB

2 Profa. Dra./Orientadora- Centro Universitário Moacyr Sreder Bastos- UniMSB, LAEFIB/FIOCRUZ, UniFOA 


\section{INTRODUÇÃO}

A regularidade na prática de atividade física e bons hábitos alimentares são idealizados como principais componentes de aspectos positivos relacionados à saúde. Segundo Silva (2011) e Valença Neto et al. (2011) pesquisando a associação entre inatividade física e obesidade entre 56 estudantes de Educação Física de uma instituição de ensino superior, no estado da Bahia, revelaram que a prática regular de atividade física reduz em 35\% o risco de morte por doenças cardiovasculares e em 33\% a mortalidade por todas as causas. É também um dos fatores que aumenta os riscos de hospitalizações e de problemas psicossociais, resultando em custos elevados para a saúde pública de modo geral. Os autores revelaram que diversos fatores, tais como: baixa disponibilidade de tempo, baixa renda familiar, companhia para a prática esportiva, aspectos demográficos e climáticos influenciam negativamente para que a prática de atividade física não aconteça.

Uma população que apresenta a renda mais baixa tende a ter uma alimentação de baixa qualidade, pouco diversificada, e hipercalórica, por vezes, por não ter condições da compra de verduras, legumes e frutas com frequência, pois são alimentos mais caros, dando preferência para o arroz, o feijão, a carne e o pão, considerados alimentos com maior quantidade de calorias (UCHIMURA, et al. 2011).

No entanto, universitários que estudam no turno noturno, e trabalham ou estagiam durante o dia possuem pouca disponibilidade de tempo para realizarem uma alimentação balanceada. Além disso, possuem também gastos com materiais do curso, mensalidade e transporte. Assim, há o aumento do consumo de alimentos com alto valor calórico, os chamados fast foods, e baixo consumo de alimentos mais nutritivos, colocando o organismo em diversas carências que podem acarretar prejuízos à saúde.

Em pesquisas para analisar características da alimentação e da atividade física, Soar, et al. (2012) investigou 61 universitários da área da saúde dos cursos de Enfermagem, Biomedicina, Farmácia, Fisioterapia, Nutrição, Odontologia e Terapia Ocupacional de uma instituição privada e foi observado que o estilo de vida deles pode contribuir para o aparecimento de doenças crônicas não transmissíveis. Portanto segundo o autor, hábitos alimentares não saudáveis e reduzida prática de atividade física durante o período da graduação frequentemente se mantêm após a formação universitária. O baixo nível de atividade física associada a hábitos alimentares não saudáveis são condições que estão relacionadas ao desenvolvimento de doenças, tais como: diabetes, infarto do miocárdio, doença arterial coronariana, câncer e obesidade.

Segundo Santos, et al. (2012), o crescente índice de obesidade presente em crianças, adolescentes e adultos no Brasil e no mundo vem alarmando a saúde pública. Nas pesquisas de Teixeira, et al. (2012) e Ramalho et al. (2012) foi observado que a mídia, principalmente a televisiva, contribui para esse índice através de comercias, anúncios em jornais, revistas, outdoors uma vez que transmitem a ideia de que aquele produto vai trazer bem estar influenciando o indivíduo a consumi-lo uma vez que o mesmo apresenta boa aparência, descartando a importância de seu valor nutricional.

Em outras pesquisas, Teixeira et al. (2012) e Ramalho et al. (2012) levantaram estudos que se complementam. Pesquisaram a prevalência da substituição do almoço e do jantar por lanches em adolescentes e a associação com estado nutricional e sexo. No entanto, Teixeira et al. (2012) verificaram a prevalência da substituição do almoço e do jantar por lanches em adolescentes e a associação com estado nutricional e sexo. Participaram 106 estudantes de uma escola técnica localizada no município de São Paulo. Já Ramalho et al. (2012) investigaram 863 universitários em 34 cursos de graduação na Universidade Federal do Acre, com o objetivo de analisar a prevalência e os fatores associados ao consumo regular de frutas e hortaliças em estudantes e abordar os possíveis motivos que os levam à troca de alimentação. Assim, identificaram maior prevalência de substituição do almoço e do jantar por lanche entre adolescentes obesos. Os substitutos do almoço e do jantar (sanduíches, salgados, pizzas e hambúrgueres) apresentavam, em sua maioria, alta densidade energética e baixo valor nutritivo, atribuindo pouca importância aos alimentos mais nutritivos como verduras, frutas e hortaliças. 
Em alguns estudos, Lanes et al. (2012) investigaram as percepções das crianças, na faixa etária entre 3 e 5 anos, da rede particular de ensino da cidade de Uruguaiana/RS, acerca dos hábitos alimentares saudáveis. Avaliaram também a eficácia da utilização do lúdico com recurso pedagógico durante as aulas de Educação Física na Educação Infantil. Os autores observaram que as crianças antes da intervenção não possuíam conhecimento sobre alimentação saudável e depois da intervenção foram capazes de identificar através de figuras em jornais e revistas quais alimentações eram mais saudáveis. Assim percebemos que essas intervenções são capazes de modificar positivamente a vida dos indivíduos que participam dessas propostas educativas, sejam crianças, jovens ou adultos.

Pesquisando sobre a promoção de hábitos alimentares saudáveis na escola e proposta metodológica de capacitação para educadores e donos de cantina escolares, Schmitz et al. (2008), seguindo este objetivo, utilizaram estratégias que estimulavam os participantes a terem iniciativa para questionar, descobrir e compreender a realidade escolar e a temática da alimentação saudável, com base em interações com os demais elementos do contexto histórico e social da escola na qual estão inseridos, propiciando espaços para a reestruturação de conhecimentos. O projeto já foi aplicado em mais de 95 escolas, compreendendo 9.500 alunos, capacitando 270 educadores e 60 donos de cantina escolar com adesão voluntária. Os resultados indicaram que a ampliação do conhecimento e as atividades desenvolvidas em sala de aula têm o potencial de estimular a formação de hábitos alimentares saudáveis nos escolares e na maioria dos indivíduos que compõem a comunidade escolar.

No entanto, para Cavalcanti, et al. (2011) a escola é um local privilegiado, onde o estudante permanece um importante período do seu tempo, pois estabelece suas primeiras interações e relações sociais, ocorrendo experiências promovedoras à construção de valores e podendo adquirir hábitos de vida saudáveis, tendo em vista também o seu papel no desenvolvimento e aprendizagem para formação social do cidadão. No entanto, para Feitosa et al. (2010) é necessário a conscientização e o reconhecimento da importância de uma alimentação saudável com a realização de programas de educação alimentar no ambiente universitário e eventos que valorizem práticas saudáveis de vida, visando fomentar melhor qualidade de vida futura.

O objetivo desse estudo é identificar o perfil nutricional dos universitários de dois cursos distintos da área da saúde: Educação Física e Fisioterapia para posterior elaboração de estratégias de ensino que viabilizem mudanças nas práticas de alimentação e de atividade física.

\section{CAMINHO METODOLÓGICO}

O presente trabalho é um estudo descritivo exploratório com universitários do curso de Educação Física e Fisioterapia do período noturno de uma instituição privada localizada na zona oeste do Estado do Rio de Janeiro.

A pesquisa foi realizada no segundo semestre de 2012, e o instrumento de coleta de dados foi um questionário aplicado em dias previamente agendados com a coordenação, durante os intervalos das aulas para que os estudantes de Educação Física e Fisioterapia pudessem respondê-lo preservando os horários das aulas. Antes da aplicação do instrumento foi realizado esclarecimentos sobre o estudo para que os interessados participassem.

Para coletar os dados foi criado um questionário objetivo com duas etapas; a primeira, contendo questões objetivas relacionadas ao perfil dos estudantes, e a segunda relacionada aos hábitos alimentares e frequência de atividade física semanal.

Esteestudoéprodutodeumprojeto submetidoàPlataforma Brasileaprovado sob onúmero 04665012600005237. 


\section{RESULTADOS E DISCUSSÃO}

Participaram da pesquisa 129 estudantes, 74 mulheres e 55 homens, a maioria com idades variando entre 18 a 32 anos, solteiros, moram com os pais, renda familiar de até cinco salários mínimo. Deslocam-se para o trabalho através de transporte coletivo, preocupam-se com a qualidade das refeições, já cursaram disciplina abordando o tema nutrição e avaliam como muito importante a disciplina que aborda a temas relacionados à nutrição.

Os dados foram coletados no segundo semestre de 2012, tabulados separadamente e posteriormente foram criados gráficos comparativos entre os cursos, para uma melhor interpretação dos dados. Os gráficos foram comparados, para se traçar o perfil dos estudantes dos cursos a respeito dos hábitos alimentares e a frequência de atividade física.

Após a análise dos dados, foram selecionados os dados mais relevantes totalizando 7 gráficos diferentes distribuídos em horas trabalhadas por dia, quantidade de atividade física realizada na semana, refeições realizadas durante o dia, consumo de verduras, frutas e grãos durante a semana, consumo de fast food, nutrientes encontrados nas refeições e consumo de bebida alcóolica durante a semana.

Com a influência da mídia e a escassez de tempo, cada vez mais pessoas tem adotado os fast foods para substituírem suas refeições. Com o objetivo de economizarem tempo aderem a este tipo de refeição por serem de rápido consumo já que na maioria das vezes não é possível realizar as refeições em casa. A consequência dessa adesão é a perda da qualidade oferecida nas refeições saudáveis enriquecidas com vitaminas, sais minerais, proteínas dentre outros nutrientes importantes para manutenção do organismo (TEIXEIRA, et al. 2012; RAMALHO et al. 2012).

Analisando os dados observamos que em relação à quantidade de horas trabalhadas durante o dia (Figura 1) foi evidenciado que 97 estudantes trabalham, representando a maioria, fato que pode ser relacionado a pouca disponibilidade de tempo para realização de atividade física e ingestão de uma refeição equilibrada.

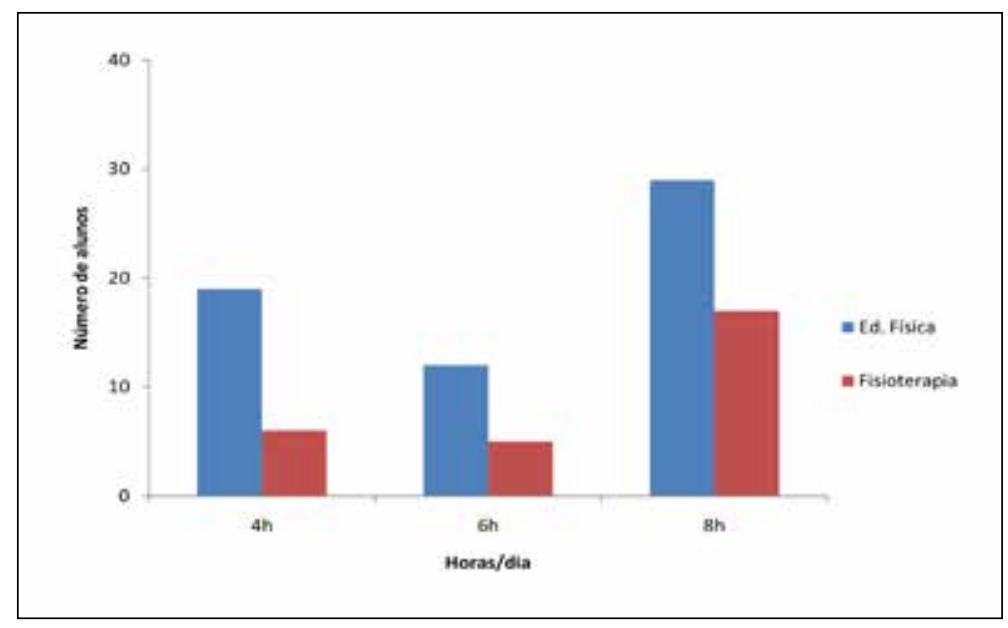

Figura 1: Quantidade de horas trabalhadas durante o dia.

Observamos que a maioria dos estudantes que responderam as perguntas do instrumento de coleta de dados (97) trabalha 8 horas por dia. Segundo Fontana e L. Brigo (2012), indivíduos que trabalham e estudam possuem menos tempo para a alimentação e atividade física. Neste caso, o ambiente de trabalho não propicia o intervalo possível para uma alimentação mais saudável devido ao tempo disponível e muitas vezes a localização dos espaços de alimentação facilitando ao trabalhador o consumo de fast foods. Este tipo de refeição pode ser repetida no intervalo entre trabalho e faculdade (FIN, et al. 2011). 
Segundo Souza et al. (2011) apesar de o Brasil apresentar algumas intervenções destinadas à promoção de atividade física e alimentação saudável em escolares, pouco se conhece sobre a metodologia dos estudos, os tipos de intervenção, as evidências dos efeitos e os resultados na saúde dos estudantes. No entanto, para Silva (2011) a sociedade acredita que estudantes do curso de Educação Física tenham hábitos mais saudáveis em seu cotidiano, sobretudo por terem disciplinas curriculares que tratam dos conceitos de educação em saúde, promoção da saúde e da importância de um estilo de vida ativo fisicamente.

Com a carga horária diária de trabalho elevada, como mostrado na figura 1, os universitários praticam menos atividade física e a qualidade das refeições ocupam o segundo plano, como indicado na figura 2, porém os dados mostram que os estudantes de Educação Física, apesar de também trabalharem 8 horas diárias conseguem ter uma boa alimentação e praticar atividade física regularmente.

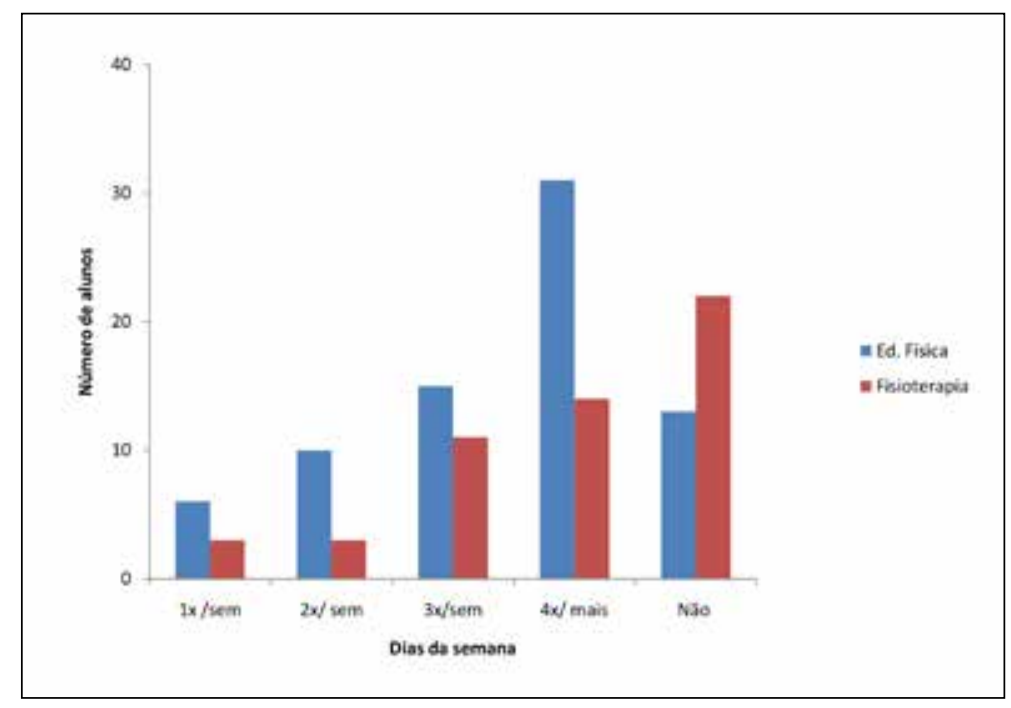

Figura 2: Relação de atividade física por semana.

Na figura 2 em relação à frequência de atividade física semanal, os dados revelam que a maioria dos estudantes pesquisados do curso de Educação Física realiza atividade física 4 vezes ou mais durante a semana, enquanto a maioria dos estudantes pesquisados do curso de Fisioterapia não realiza atividade física regularmente, concordando com Silva (2011) que revelou em sua pesquisa que estudantes da área de saúde praticam pouca atividade física, porém os achados dos estudantes de Educação Física neste trabalho são relevantes, visto que foi apontado nos dados a boa frequência de realização de atividade física.

Diversos fatores podem influenciar na diminuição ou ausência da prática regular de atividade física, como pouca disponibilidade de tempo, dinheiro, companhia para a atividade física, limitações ambientais como condições climáticas desfavoráveis como chuva constante e calor excessivo. Todos estes fatores contribuem para que o indivíduo se afaste da atividade física e como consequência causando malefícios à saúde (ABBES, et al. 2011).

Em estudos Valença Neto et al. (2011) avaliando a associação entre a inatividade física e obesidade entre 56 estudantes do curso de Educação Física de uma instituição de ensino superior do estado da Bahia observou que o baixo nível de atividade física, está associado ao desenvolvimento de doenças, tais como: diabetes, infarto do miocárdio, doença arterial coronariana, câncer e obesidade, doenças essas que podem aumentar o índice de hospitalizações e gastos com a saúde pública. O que se torna um fator de risco aos estudantes do curso de Fisioterapia, presentes neste trabalho, por apresentarem baixo índice de frequência de atividade física semanal.

Já os estudantes do curso de Educação Física são beneficiados por praticarem atividade física regularmente, pois segundo Silva (2011) é bem relatada em estudos recentes que a prática regular de atividade física 
reduz em 35\% o risco de morte por doenças cardiovasculares e em $33 \%$ a mortalidade por todas as causas. Neste sentido, deve-se estimular continuamente que as pessoas sejam ativas fisicamente em todas as etapas da vida.

O baixo índice ou a prática regular de atividade física (figura 2) pode desencadear problemas ou benefícios quando associados à quantidade adequada de refeições realizadas ao longo do dia, representado na figura 3.

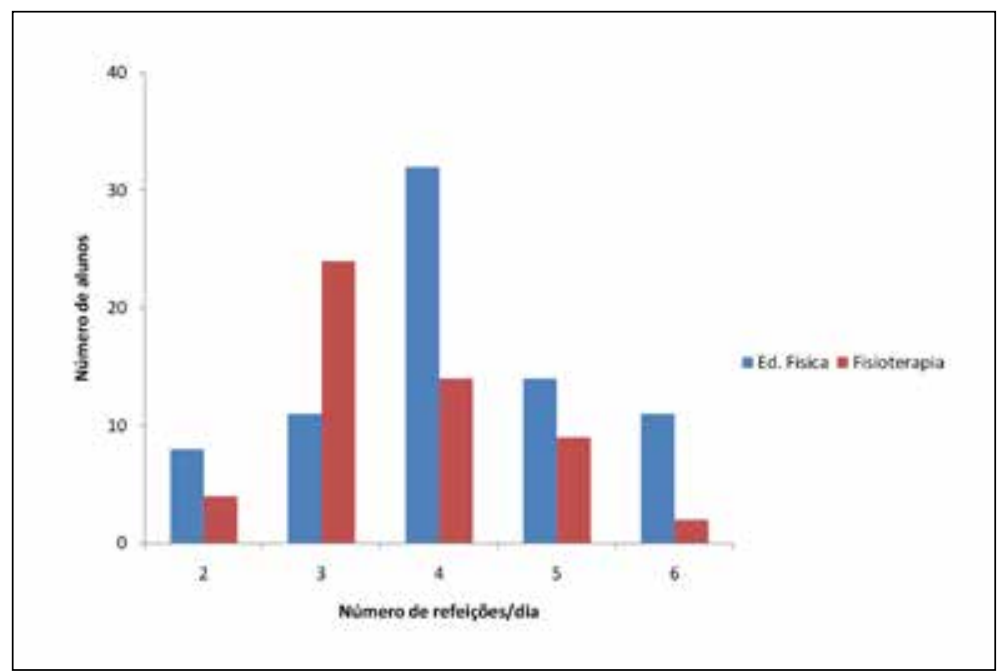

Figura 3: Quantidade de refeições realizadas ao longo do dia.

Na figura 3, os dados revelam que a maioria dos estudantes do curso de Educação Física realiza 4 refeições durante o dia atendendo ao café da manhã, almoço, lanche e jantar enquanto a maioria dos entrevistados do curso de Fisioterapia possuem o hábito de se alimentar apenas 3 vezes durante o dia.

Os estudos de Teixeira et al. (2012) e Ramalho et al. (2012) também nos mostram que cada vez mais pessoas vêm substituindo o almoço e o jantar por fast foods. Alguns dos motivos apontados para essas adesões são: praticidade, variedade de lanchonetes e restaurantes, influência de amigos e da mídia. Sugerimos que esses fatos estão associados à menor quantidade de refeições realizadas durante o dia por estudantes de Fisioterapia quando comparados aos estudantes de Educação Física.

Um fato relevante em relação à diferença de refeições realizadas durante o dia por universitários de ambos os cursos é que estudantes de Educação Física se preocupam com a imagem corporal, o que torna necessário ter uma alimentação saudável e balanceada incluindo variedade e quantidade de alimentos diários, segundo Silva et al. (2011). O mesmo parece não ocorrer com os estudantes de Fisioterapia uma vez que praticam menos atividade física e realizam menos refeições ao longo do dia.

A maioria dos estudantes de ambos os cursos realizam 3 a 4 refeições ao longo do dia, como apresentado na figura 3, e consomem verduras, frutas e grãos apenas 3 vezes na semana (figura 4) ou seja, a frequência do consumo desses alimentos semanalmente é um pouco menor do que a quantidade de refeições, concluindo que em alguns dias da semana, eles não estão presentes no cardápio. De um modo geral observamos que tanto os alunos de um curso quanto do outro não fazem as 6 refeições indicadas pelo Ministério da Saúde (2006), que estabelece-se pelo menos 3 refeições (café da manhã, almoço e jantar) e 3 lanches saudáveis por dia, sem pular as refeições. 


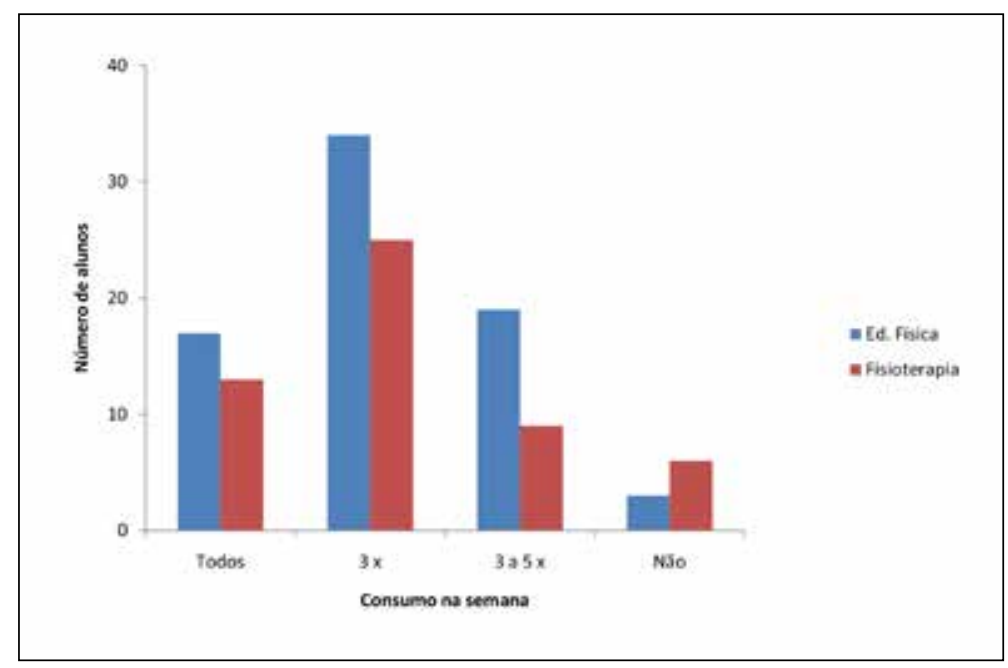

Figura 4: Consumo de verduras, frutas e grãos durante a semana.

Os dados mostram na figura 4 que a maioria dos estudantes que responderam a essa pergunta no questionário, representado por 34 estudantes de Educação Física e 25 de Fisioterapia possuem o hábito de consumir verduras, frutas e grãos 3 vezes na semana. Para Ramalho et al. (2012) o acesso adequado a esses alimentos é importante para a saúde por serem ricos em micronutrientes e terem baixa densidade energética. Além disso, são alimentos protetores para várias doenças crônicas não transmissíveis. Os estudos de Ramalho, et al. (2011) revelam a direta ligação de doenças cardiovasculares e o câncer com a insuficiência do consumo de hortaliças e frutas.

Conforme as informações contidas no gráfico, é possível notar que o curso de Fisioterapia, mesmo tendo presente em seu cardápio as verduras, frutas e grãos, comparado ao curso de Educação Física, a ingestão é menor. Isso pode ser decorrente ao pouco tempo disponível dedicado a uma refeição balanceada, pois a maioria possui grande carga horária de trabalho (figura 1), além de outras variáveis a serem consideradas, não presentes na atual pesquisa. O que pode causar malefícios à saúde, visto que os estudantes de Fisioterapia não possuem o hábito de praticar atividade física frequentemente, como mostrado na figura 2.

Em relação ao consumo de Fast foods ambos os curso apresentam baixo consumo de tais alimentos, como evidenciado na figura 5, um fato relevante, visto que estudantes de Fisioterapia, mesmo tendo pouco tempo para uma alimentação balanceada, não possuem o hábito de consumir alimentos de rápido preparo.

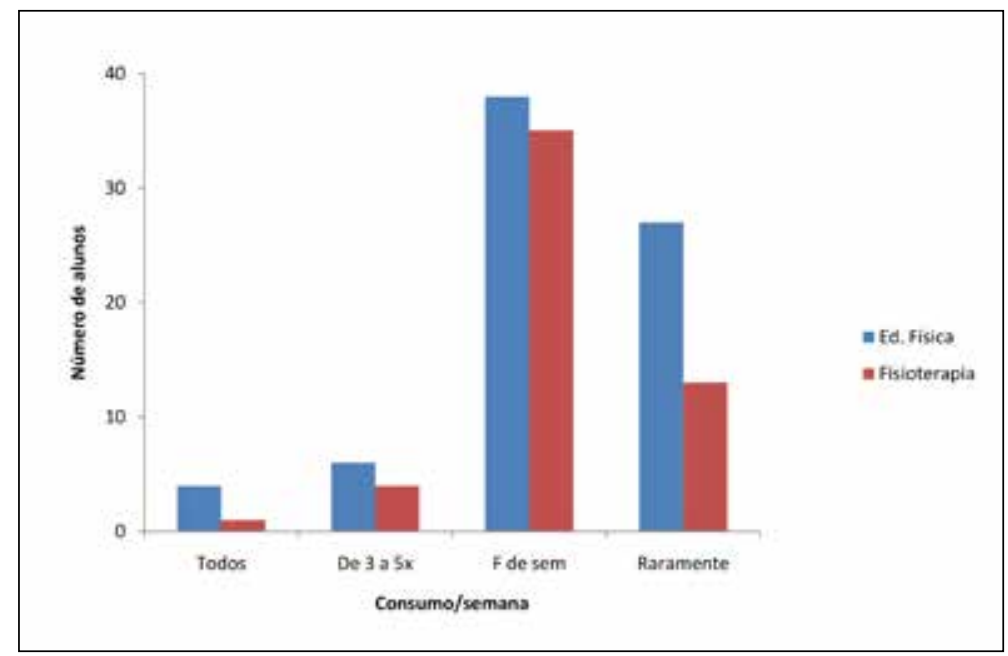

Figura 5: Quantidade de fast foods consumida durante a semana. 
Foi observado na figura 5, que a maioria dos estudantes (73) pesquisados nos dois cursos consome fast foods apenas nos finais de semana. O consumo de alimentos altamente calóricos e baixo valor nutricional, ricos em açúcares e gorduras em elevadas quantidades acarretam diversos malefícios ao indivíduo, tais como obesidade e doenças cardiovasculares conforme relatam Teixeira et al. (2012) e Maciel et al. (2012 ). Porém como já observado a quantidade de refeições ao longo do dia é reduzida em universitários do curso de Fisioterapia, representando apenas 3. Assim, mostra que apesar de serem estudantes da área da saúde e possuírem disciplinas curriculares relacionadas à nutrição possuem hábitos alimentares inadequados.

É recomendado evitar a substituição de refeições essenciais no dia-a-dia por lanches rápidos, pois esses reduzem a ingestão de alimentos tradicionais, que são fontes importantes de vitaminas minerais e fibras, o que pode acarretar problemas de saúde futuros quando há essa substituição por alimentos de alta densidade energética (RAMALHO et al. 2012; TEIXEIRA et al. 2012).

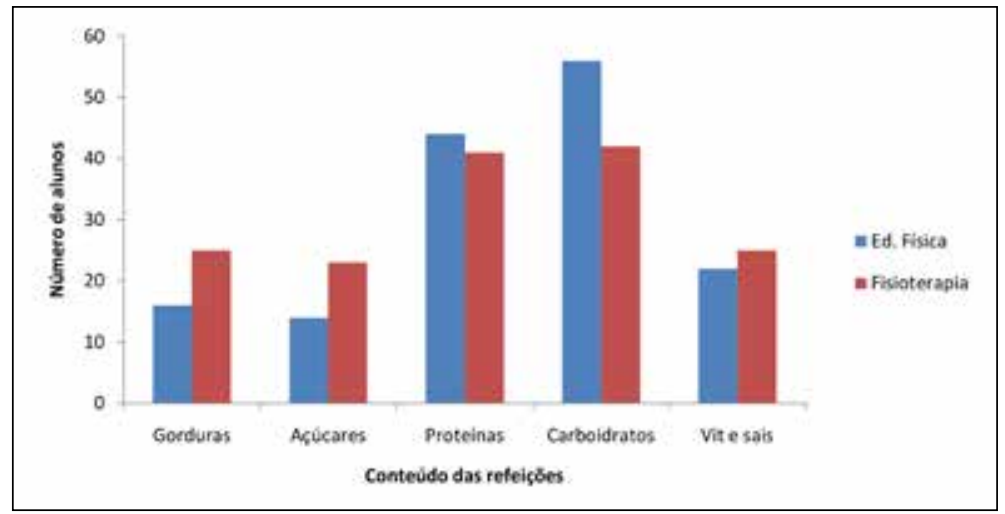

Figura 6: Conteúdo das refeições diárias dos universitários.

Quando perguntado aos estudantes sobre o conteúdo presente em suas refeições, a Figura 6 evidencia que a maioria dos universitários pesquisados consome todas as opções oferecidas, porém em maior quantidade para proteínas e carboidratos, principalmente para os estudantes do curso de Educação Física. Os níveis de gorduras e açúcares também não são baixos a ponto de serem ignorados, pois comparados ao número de estudantes que participaram da pesquisa é um valor que deve ser considerado. Assim, gorduras e açúcares quando consumidos em quantidades superiores ao que o organismo necessita causa malefícios a saúde como diabetes, hipertensão e obesidade. Hábitos alimentares não saudáveis e reduzida prática de atividade física durante o período da graduação, em alguns indivíduos, se mantêm após a formação universitária, seja por pouca disponibilidade de tempo, por não terem uma educação nutricional correta, por cultura familiar ou por baixa renda mensal. A dieta denominada ocidental, rica em gorduras, açúcares simples, alimentos refinados e reduzidos em fibras, bem como a inatividade física, fazem parte do estilo de vida de grande parte da população (SOAR, et al. 2012; MACIEL, et al. 2012) o que compromete o estado de saúde do indivíduo.

Com base nos dados acima, é possível observar que os universitários possuem o conhecimento sobre a composição de suas refeições e a importância da presença de elemento em seu cardápio. Sugere-se que isso ocorra devido à base do conteúdo sobre nutrição abordado nas disciplinas encontradas na grade curricular, uma vez que, a maioria dos estudantes relatou já ter cursado disciplinas que abordam esse conteúdo durante a graduação.

Quanto ao perfil dos estudantes observamos que a maioria dos universitários dos cursos investigados consome pouco fast foods apesar do pouco tempo dedicado às refeições. Sugere-se que o fato de a maioria ser solteira e morar com os pais, conseguem incluir em suas alimentações as verduras, frutas, hortaliças e grãos. A 
alimentação balanceada pode ser atribuída à cultura familiar e o zelo dos pais quanto aos sentidos importantes do consumo de nutrientes essenciais à boa saúde (RAMALHO et al. 2012 e MACIEL et al. 2012).

A cultura familiar também pode ser associada em alguns casos, à ingestão de alimentos mais saudáveis, com maior valor nutricional e sugerindo também o não consumo de bebida alcóolica, como representado na figura 7 .

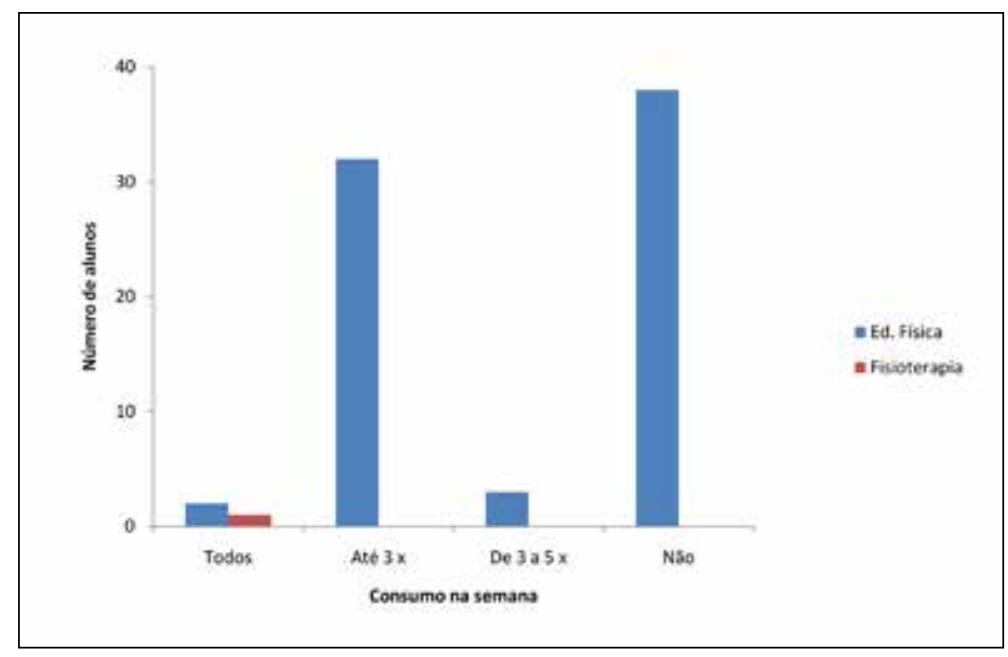

Figura 7: Consumo de bebida alcoólica durante a semana.

Em relação à questão sobre a frequência do consumo de bebida alcoólica a figura 7 nos mostra que a maioria dos estudantes declarou não consumir. Este dado é um ponto positivo, pois, segundo Lepre e Martins (2009) em uma pesquisa realizada com estudantes do ensino médio de uma escola pública a respeito das consequências do consumo do álcool sobre a vida escolar, o uso abusivo do álcool faz com que os estudantes apresentem queda no desempenho escolar, sonolência, lentidão e dificuldade para entender o que o professor diz em sala de aula. Portanto, estudantes que não possuem o hábito de consumir álcool, tendem a ter mais disposição para realização de atividade física, e menores probabilidades de consumo de alimentos altamente calóricos e com pouco valor nutricional, sugerindo que esses estudantes também possuem mais atenção durante as aulas.

\section{CONCLUSÃO}

A alimentação balanceada associada à prática de atividade física proporciona múltiplos benefícios à saúde, reduzindo a probabilidade de se adquirir doenças como obesidade e hipertensão. Conclui-se que os estudantes do curso de Educação Física possuem uma boa educação alimentar e conscientização sobre benefícios da prática de atividade física, como consequência aderem hábitos alimentares saudáveis consumindo pouco fast foods e mais proteínas, carboidratos, vitaminas e sais minerais em quantidades capazes de suprirem as necessidades exigidas pelo organismo. Assim, possuem menos probabilidade de desenvolverem doenças crônicas não transmissíveis uma vez que a alimentação da maioria dos estudantes está associada à prática de atividade física. Entretanto, a preocupação com a imagem corporal está altamente relacionada com a boa qualidade na alimentação e prática frequente de atividade física. Já os estudantes de Fisioterapia, apresentaram hábitos inadequados tanto na alimentação quanto na prática de atividade física, tendo maior probabilidade de se tornarem indivíduos obesos devido à falta de atividade física regular. Fato que deve ser estudo com mais afinco, visto que esses futuros profissionais serão responsáveis pelo bem estar e qualidade de vida de seus futuros pacientes. 
No entanto, mais estudos são necessários para traçar o perfil alimentar de estudantes universitários da área de saúde, uma vez que o número de estudantes pesquisados não é suficiente para concluir o perfil dos estudantes desta área. Os dados deste estudo podem contribuir para a elaboração e desenvolvimentos de projetos dentro das instituições de ensino superior voltados para o incentivo do consumo da alimentação balanceada e da prática de atividade física entre os universitários, além de incentivar a elaboração e aplicação de estratégias diversificadas entre os professores de disciplinas específicas relacionadas ao tema no decorrer do curso. Este pode ser um pequeno passo para que esses indivíduos mudem seus hábitos tanto em relação ao consumo de alimentos de alta densidade energética quanto da prática de atividade física.

\section{AGRADECIMENTOS}

Ao Centro Universitário Moacyr Sreder Bastos/UniMSB, pelo apoio financeiro oferecido à essa pesquisa.

\section{REFERÊNCIAS}

ABBES, Priscila Trapp; LAVRADOR, Maria Silvia Ferrari; ESCRIVÃO, Maria Arlete Meil Schimith; et al. Sedentarismo e variáveis clínico-metabólicas associadas à obesidade em adolescentes. Revista de Nutrição, Campinas, v.24, n.4, p.529-538, Jul/Ago., 2011.

CAVALCANTI, Leonardo A.; CARMO JUNIOR, Tugdual R. do; PEREIRA, Lílian A.; ASANO, Ricardo Y.; GARCIA, Márcia C. L.; CARDEAL, Cintia M.; FRANÇA, Nanci M. de. Efeitos de uma intervenção em escolares do ensino fundamental, para a promoção de hábitos alimentares saudáveis. Revista Brasileira de Ciência e Movimento, v.20, n.2, p.5-13, 2011.

FEITOSA, Elaine Prado Santos; DANTAS, Camila Andrade de Oliveira; WARTHA, Elma Regina Silva Andrade; et al. Hábitos alimentares de estudantes de uma universidade pública no Nordeste, Brasil. Revista Alim. Nutr., Araraquara, v.21, n.2, p.225-230, Abr/Jun, 2010.

FIN, Gracielle; BARETTA, Elisabeth; NODARI JÚNIOR, Rudy José. Nível de atividade física, hábitos alimentares e indicadores antropométricos de funcionários da universidade do oeste de Santa Catarina. Evidência, Joaçaba v.11 n.2, p.57-68, Julho/Dezembro, 2011.

LANES, Dário Vinícius Ceccon; SANTOS Marcelli Evans Telles dos; SILVA, Elane Fabíola de Sousa Jerônimo; et al. Estratégias lúdicas para a construção de hábitos alimentares saudáveis na educação infantil. Revista Ciências \& Ideias, v.4, n. 1, Julho, 2012.

LEPRE, Rita Melissa; MARTINS, Raul Aragão. Raciocínio moral e uso abusivo de bebidas alcoólicas por adolescentes. Paideia, São Paulo, v. 19, n. 42, p.39-45, Jan/Abr, 2009. SILVA, Diego Augusto Santos. Nível de atividade física e fatores associados em acadêmicos de educação física de uma universidade pública do nordeste do Brasil. Revista Brasileira de Atividade Física \& Saúde, v.16, n.3, p.193-198, Jan/Fev, 2011.

MACIEL, Erika da Silva; SONATI, Jaqueline Girnos; MODENEZE Denis Marcelo; et al. Revista de Nutrição, Campinas, v.25, n.6, p.707-718, nov./dez., 2012.

RAMALHO, Alanderson Alves; DALAMARIA, Tatiane; SOUZA, Orivaldo Florencio de. Consumo regular de frutas e hortaliças por estudantes universitários em Rio Branco, Acre, Brasil: prevalência e fatores associados. Caderno de Saúde Pública, Rio de Janeiro, v.28, n.7, p.1405-1443, Jul, 2012.

SANTOS, Cíntia da Conceição; STUCHI, Rosamary Aparecida Garcia; SENA, Cristina Arreguy; et al. A Influência da Televisão nos Hábitos, Costumes e Comportamento Alimentar. Cogitare Enferm. V.17, n.1, p.65-71, Jan/Mar, 2012. 
SCHUMITZ, Bethsaída de Abreu Soares; RECINE, Elisabetta; CARDOSO, Gabriela Tavares; et al. A escola promovendo hábitos alimentares saudáveis: uma proposta metodológicas de capacitação para educadores e donos de cantina escolar. Caderno de Saúde Pública, Rio de Janeiro, v. 24, n. 2, p. 312-322.

SILVA, Tatiana Rodrigues da; SAENGER, Guilherme; PEREIRA, Érico Felden. Fatores associados à imagem corporal em estudantes de Educação Física. Motriz, Rio Claro, v.17, n.4, p.630-639, Out./Dez, 2011.

SOAR, Claudia; SILVA, Patrícia de Souza e; LIRA, Janaína Guarino. Consumo alimentar e atividade física de estudantes universitários da área de saúde. Revista Univap, São José dos Campos-SP, v.18, n.31, p.41-47, Jun, 2012.

SOUZA, Evanice Avelino de; BARBOSA FILHO, Valter Cordeiro; NOGUEIRA, Júlia Aparecida Devidé; et al., Atividade física e alimentação saudável em escolares brasileiros: revisão de programas de intervenção. Caderno de Saúde Pública, Rio de Janeiro, v.27, n.8, p.1459-1471, Ago, 2011.

TEIXEIRA, Amanda Solimani; PHILIPPI, Sonia Tucunduva; LEAL, Greisse Viero da S.; et al. Substituição de refeições por lanches em adolescentes. Revista Paul Pediatr, v.30, n. 3, p.330-337, 2012.

VALENÇA NETO, Paulo Fonseca; AMARAL, Carlos Silva; NOGUEIRA, Thiago Marques; ROCHA, Saulo Vasconcelos. Inatividade física e obesidade central entre estudantes de educação física de uma instituição de ensino superior. Revista Brasileira de Obesidade, Nutrição e Emagrecimento, São Paulo, v.5, p.162-168, Maio/Jun, 2011.

Ministério da Saúde. Alimentação saudável para todos: Siga os dez passos. Disponível em: <http://nutricao.saude.gov.br/>. Acesso em: 26/03/2013. 